\title{
BIM Integrated LCA for Promoting Circular Economy towards Sustainable Construction: An Analytical Review
}

\author{
Kai Xue 1,2,3 ${ }^{\mathbb{D}}$, Md. Uzzal Hossain ${ }^{2,4}$, Meng Liu 1,2, ${ }^{\text {, Mingjun Ma }}{ }^{1,2,5}$, Yizhi Zhang ${ }^{1,2}$, Mengqiang Hu ${ }^{1,2}$, \\ XiaoYi Chen ${ }^{1,2}$ and Guangyu Cao ${ }^{3}$
}

1 School of Civil Engineering, Chongqing University, Chongqing 400045, China; xuek1213@126.com (K.X.); mamingjuncqjg@163.com (M.M.); 20145465@cqu.edu.cn (Y.Z.); mengqiang94@126.com (M.H.); chenxiaoyivip@163.com (X.C.)

2 National Centre for International Research of Low-Carbon and Green Buildings (Ministry of Science and Technology), Chongqing University, Chongqing 400045, China; uzzal@hku.hk

3 Department of Energy and Process Engineering, Norwegian University of Science and Technology (NTNU), 7014 Trondheim, Norway; guangyu.cao@ntnu.no

4 Department of Civil Engineering, The University of Hong Kong, Hong Kong 999077, China

5 Chongqing Construction Engineering Group Corporation Limited, Chongqing 401122, China

* Correspondence: liumeng2033@126.com

Citation: Xue, K.; Hossain, M.U.; Liu, M.; Ma, M.; Zhang, Y.; Hu, M.; Chen, X.; Cao, G. BIM Integrated LCA for Promoting Circular Economy towards Sustainable Construction: An Analytical Review. Sustainability 2021, 13, 1310. https://doi.org/ $10.3390 /$ su13031310

Academic Editor: Antonio Caggiano Received: 31 December 2020

Accepted: 24 January 2021

Published: 27 January 2021

Publisher's Note: MDPI stays neutral with regard to jurisdictional claims in published maps and institutional affiliations.

Copyright: (c) 2021 by the authors. Licensee MDPI, Basel, Switzerland. This article is an open access article distributed under the terms and conditions of the Creative Commons Attribution (CC BY) license (https:/ / creativecommons.org/licenses/by/ $4.0 /)$.

\begin{abstract}
Sustainable construction is widely regarded as one of the best decisions to reduce the environmental impact of the buildings. Numerous scientific efforts have been devoted to promoting sustainability by adopting different techniques such as life cycle assessment (LCA) and building information modeling (BIM). The recent circular economy (CE) agenda is prioritizing in different sectors including construction due to increasing concern on the materials efficiency and resources consumption. Therefore, this study aimed to identify the implications, considerations, contributions and challenges of BIM integrated LCA and the CE adoption in the industry by critically analyzing the recent literature. A scientometric analysis was applied to the selected studies to show the knowledge among BIM, LCA and CE relationships. With a comprehensive analysis of major challenges and opportunities of LCA and BIM for construction sustainability, key drivers for sustainable building design were identified and analyzed. A prospective integrated framework for CE adoption into BIM-based LCA of buildings and an evaluation method are proposed by analyzing the contemporary issues. It is believed that the analyzed critical issues, identified future research direction and proposed frameworks and this methodology should contribute to effective implementation of CE into the building for promoting sustainable construction.
\end{abstract}

Keywords: building; building information modeling; circular economy; sustainable construction; life cycle assessment; sustainability

\section{Introduction}

The construction industry, including building, is one of the main factors leading to environmental degradation, resources consumption and climate change impacts because it accounts for more than $40 \%$ of the worldwide carbon emission [1,2]. Studies confirm that the construction industry is responsible for inefficient resources management due to industry-specific features such as lack of integration, cooperation and waste resources [3]. Traditional construction needs mainly the knowledge of multi-disciplinary to be superimposed. The aspects of sustainable design involved building orientation, building massing, day lighting analysis, water harvesting, energy modeling, sustainable materials and site and logistics management, which needs a smooth cooperation and comprehensive coordination to achieve a resources and time saving. Thus, the industry needs to integrate new strategies for buildings to decrease burdens on the environment [4], as well as to achieve more sustainable performance $[5,6]$. 
Globally, the emphasis on sustainable construction is widely given as priority to enhance resource efficiency through optimizing design, materials recycling and reuse and reducing environmental impact of the buildings [7], with high level social and economic development [8]. Along the design tools, life cycle assessment (LCA) technique is now widely used for providing a comprehensive environmental evaluation of products, processes and even services based on their life cycle [9]. The traditional LCA method is inherently used methodology for assessing the building's lifecycle, starting from its raw material extraction, processing, use and then to end-of-life stage [10], while with the deepening of sustainable concept, the recycling of construction resources become popular among the policymakers, academia and industry. It was recommended to access the energy consumption and subsequent environmental emissions depending on the scope for each type of building including each stage of its lifecycle [11]. LCA can assist in material selection through the sustainable sourcing of materials [12], and can also be used to predict building environmental performance, as well as sustainable decision making [13].

However, conducting LCA in buildings is complicated due to the collection of huge amounts of information which is often time-consuming [14]. After establishing the bill of quantities and finding out the representative datasets for the building materials from a database, LCA is typically conducted at the end of the design process, leading to affect the least decision-making process [15]. However, it may not be successfully used as a decision support tool in the late design stage as the changes of design or the materials selection is difficult and too costly [16]. Thus, significant impact reduction could be possible by selecting sustainable materials/processes at early design choice [17-19]. Consequently, LCA is increasingly adopted in the building design process to provide design strategies and monitor the influence of such designs. At this stage, the designers can gather the required data for LCA and can conduct initial evaluation.

Nowadays, building information modeling (BIM) is widely used in the construction industry due to the ability to modify the errors in the designs, the selection of materials, etc., at the early stages. In addition, it can effectively use in buildings' scheduling and sequencing, consulting design alternatives, assisting in the selection of most viable strategies, etc. [20]. In addition, it can enable the collaboration and communication among project participants to well-perform building construction [21]. The integrated BIM has a huge potential for achieving sustainability in the construction industry through materials selection, waste minimization, energy-saving alternatives, cost estimations, green buildings design and interoperability [3], which can also support complex decision-making process [22]. Due to its efficiency, it is also now widely used to green building evaluation process [23], improve the project information flow and achieve better performance and quality [7]. Most importantly, it can reduce the complexity of data collection required for LCA as it allows to perform quick quantity take-off $[24,25]$. Thus, the advantages mentioned above can bring a more correct and reasonable plan for decision-makers to evaluate environmental impacts in the building industry, although there is a lack of interoperability, supporting technologies and information exchange framework with BIM [3,26].

Due to resources consumption and associated environmental concern, the adoption of circular economy (CE) principles into the building industry has emerged recently. CE is deeply rooted within industrial ecology, often considered as branch of industrial ecology, became more and more important due to the latest environmental-socio-political agenda [27]. CE and sustainability, the interconnected concepts, are gaining popularity among the policymakers, academia and industry [28]. The key aims of CE in the building are: (i) to improve materials efficiency and optimize energy use by considering the sustainability of materials sourcing through integrating collaboration advantages between industries [29,30], (ii) to promote the maximum reusing of materials and to minimize waste generation [31], (iii) to avoid waste generation to landfill [32], (iv) to optimize economic development with the resources use and environmental impacts [33-35]. Thus, CE provides an opportunity to reduce the use of natural materials, conserving resources and reducing the carbon footprint [34] and reducing the risk of material supply and price volatility [35]. 
To ensure effective CE adoption, the performance evaluation including the quantification of building materials and components including their circularity at the early design stage is needed. Thus, based on the early stage of design, BIM can help identify material flows at different stages including the end-of-life of buildings [29], where LCA can be used to evaluate the environmental performance by included all processes from the cradle-tocradle system boundary $[31,36,37]$, for adopting the CE principle. Although several studies have focused on BIM integrated LCA for buildings, how to use the BIM-LCA platform effectively to enhance the building sustainability driven by $\mathrm{CE}$ is still a research question. By reviewing and analyzing the existing literature comprehensively, this paper aimed to explore the key drivers including the challenges and opportunities for adopting BIM integrated LCA in designing sustainable buildings, and the way of CE promotion in such integrated design by highlighting the drawbacks with potential solutions and prospective integrative framework for sustainable buildings construction.

\section{Methodology}

This study aimed to provide a comprehensive overview of BIM-LCA adoption in the building industry by a multidisciplinary approach for promoting $\mathrm{CE}$ to enhance and improve the sustainability of buildings based on a systematic review process (illustrated in Figure 1). Three established databases, such as Google Scholar, ScienceDirect and Web of Science, were used to search the relevant literature using several predefined keywords such as 'building information modeling', 'BIM', 'life cycle assessment', 'LCA', 'sustainability', 'circular economy', 'building' and 'construction'. The search strategy yielded nearly 200 literatures for the recent 20 years. However, only a few literatures were found when 'sustainability', 'circular economy' and 'BIM' were used, indicating the scope for further research in sustainable building area for promoting CE. In addition, a cross-reference investigation was conducted as non-systematic retrieval to include additional related literature.

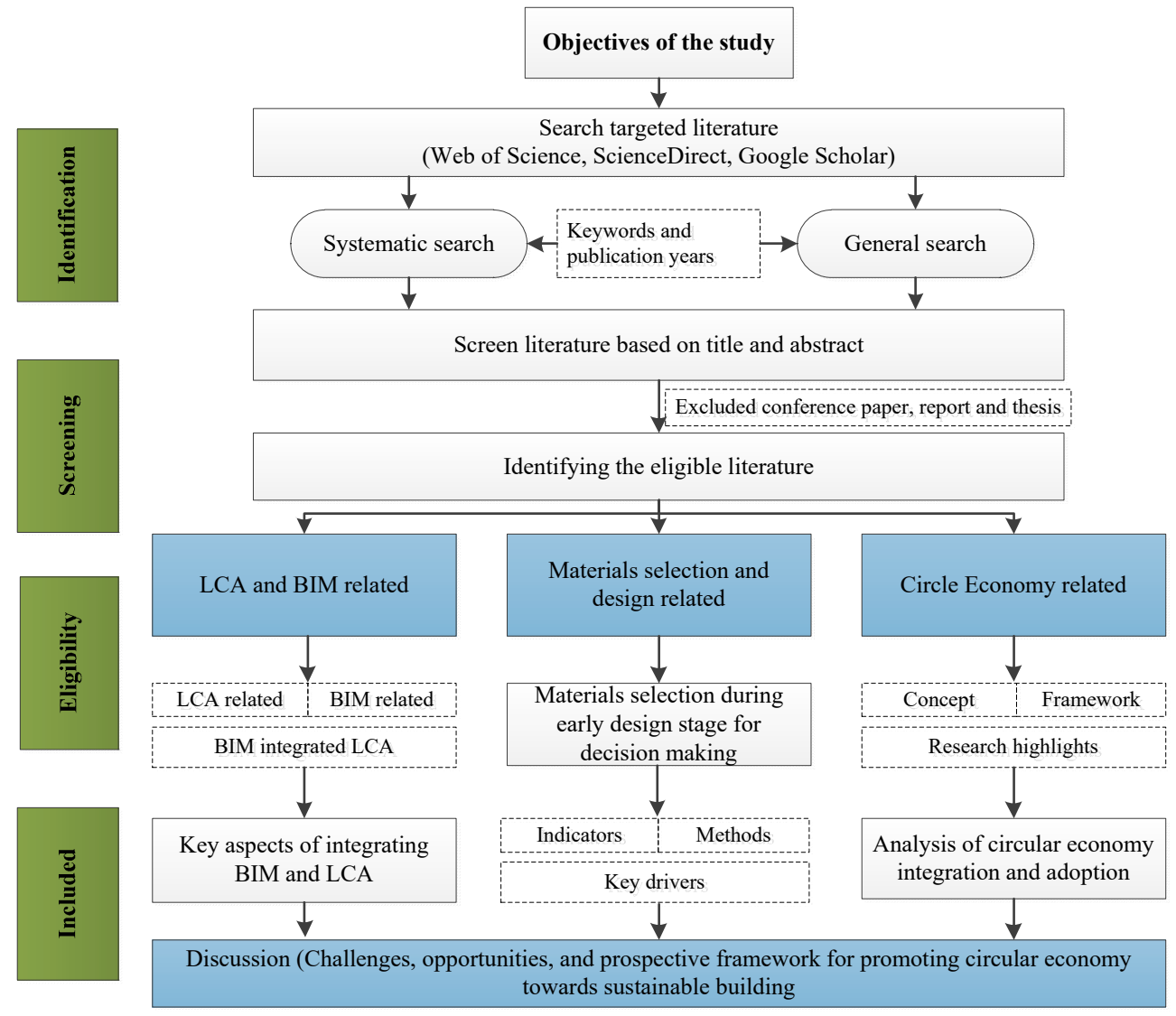

Figure 1. Outline of the research methods. 
After identifying the relevant literature, a filtration in a view of titles and abstracts was conducted to identify the closely relevant papers. Due to the availability of a large number of scientific papers published in the peer-reviewed journals, papers that were published in conferences, theses and books were eliminated. Further assessment of eligibility based on research points led to 74 studies being choose for in-depth analysis (49 were focused on BIM, LCA, material selection, and 25 were on CE). Finally, an effective comparison of results, key drivers and challenges were applied, and future research orientation was identified based on adopting CE effectively and promoting and evaluating sustainable building based on BIM-LCA platform comprehensively.

Due to increasing concern, the number of scientific contributions in building environmental research is also increasing significantly [32]. In order to analyze and visualize the bibliometric network, the scientometric analysis by a text-mining tool named VOSViewer was adopted in this study. The tool is used to analyze the distance-based visualizations of bibliometric networks, where the distance between two nodes indicates the correlation between them. The present analysis used the function of co-occurrence in VOSViewer, including keywords and locations. As keywords of existing studies depict the topics within a given domain, the network analysis of keywords demonstrates the knowledge between their interrelationships and intellectual organization of the specific studying goals. An example of the keywords network analysis within the scope of the study is shown in Figure 2.

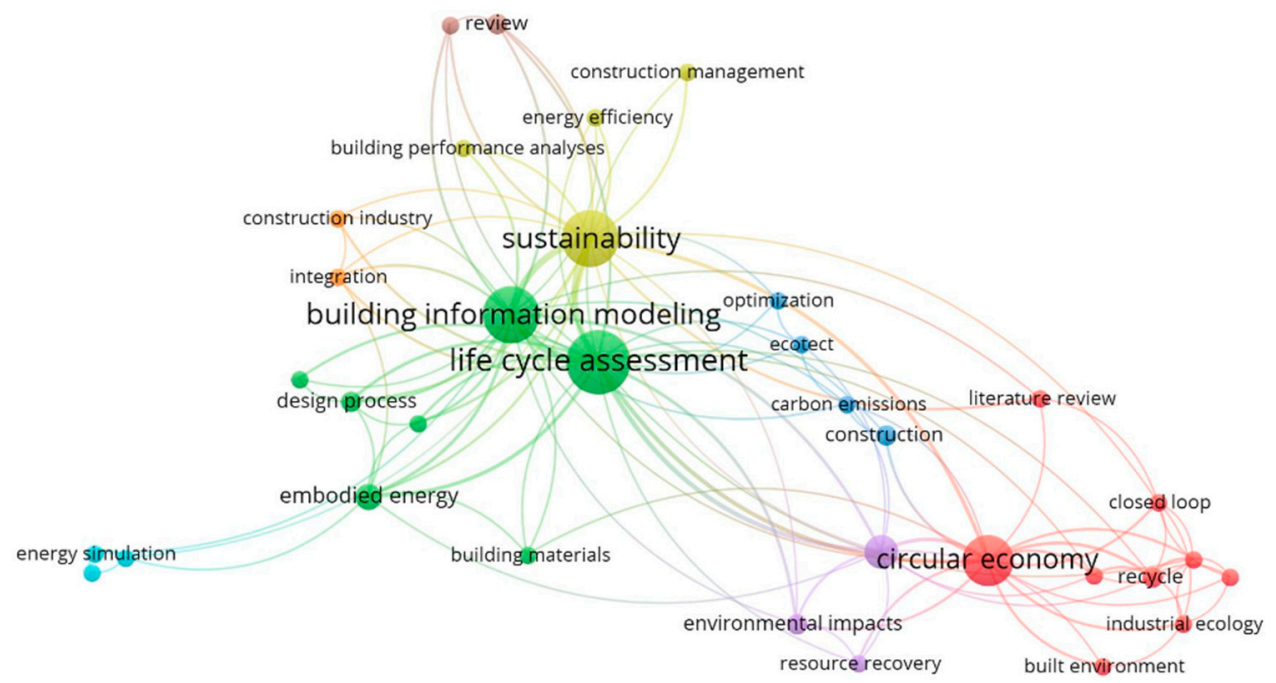

Figure 2. Co-occurrence of keywords for the selected papers.

The keywords were divided into different clusters using different colors of nodes. It can be found that the focus of this study, for instance, BIM was closely connected to LCA in the same cluster (green). Meanwhile, keywords from different categories are also closely connected, such as BIM, LCA and CE which illuminated a good relationship through buildings (purple) and sustainability (yellow) for further analysis in the paper. In addition, VOSViewer was also used to further identify and evaluate these countries' contributions to the global research community. The mapping of the active contributions for the selected papers is shown in Figure 3. It can be found that the following countries or regions have developed the research community according to their node sizes and connection lines: China, The Netherlands, UK, USA, Germany, Germany, Sweden and so on. As a result of sustainability concerns, developed countries/regions are pioneers in implementing such research, while some developing countries like China are also investing huge capital and technology in research recently. 


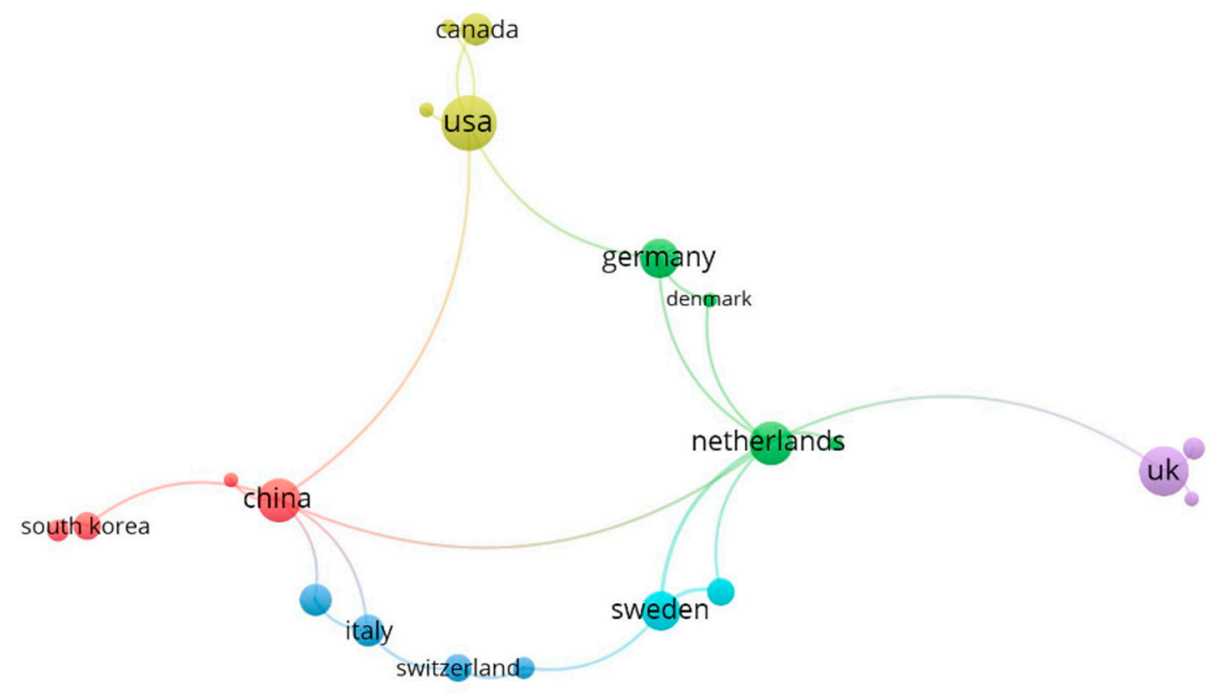

Figure 3. Mapping of countries active for the selected papers.

\section{Results and Discussion}

\subsection{Implications and Essence of the Existing Studies}

The early design and preconstruction are considered as the most critical phases to include the sustainability features in building construction as well as decision process. In this stage, different disciplines interact to offer insights and information needed for sustainable assessment, especially the quantification and selections of material [38,39]. Usually, BIM is used to design buildings and construction plans including the selection of materials whereas, LCA is used by the designers to model the design and modify where necessary through the input of required materials and energy, and then calculated the embodied impacts of their design. Thus, the integration between BIM and LCA is necessary in order to evaluate and compare the interactive abilities of different file formats between these plug-ins. For designers, it is very important to transfer conceptual information directly from the BIM model to the evaluation tool (e.g., energy analysis software) [21].

As BIM can effectively reduce the difficulty of obtaining construction data, LCA can be used to provide reaction on the environmental impacts in early-stage decision-making on different design choices [11]. A few studies demonstrated that BIM can significantly reduce the data input for the LCA application [7,40]. For example, Ajayi et al. [24] highlighted the complex and time-wasting nature of collecting and compiling the LCI. The study also suggested that the integration of BIM and LCA software may be an effective solution due to available data on the design and materials included in BIM platform. Basbagill et al. [41] developed time-reduction data acquisition strategies to assess a residential building in the USA and quantify building materials. This way rapidly enables the integration of LCA into the early stages and help designers predict which strategies can more effectively determine the specific impact of the building. Aoife et al. [25] pointed out that when working with BIM, LCA is easy to visualize the material inputs, which assist the understanding of the inputs needed and identify possible mistakes between BIM and the drawings.

Some of the selected BIM-based LCA studies with their implications are highlighted in Table 1, and different considerations such as materials used, evaluation method, indicators, etc. in the selected studies are highlighted in Table 2. It can be seen that multiple impact categories were considered in few studies [42,43], whereas most of them were focused on only one or two impacts (e.g., carbon emission and/or energy consumption) [22,26]. 
Table 1. Summary of the selected existing building information modeling (BIM) integrated life cycle assessment (LCA) studies.

\begin{tabular}{|c|c|c|}
\hline Authors & Study Highlights & Implications \\
\hline $\begin{array}{l}\text { Soust- } \\
\text { Verdaguer } \\
\text { et al. [7] }\end{array}$ & $\begin{array}{l}\text { - Analyzed methodological aspects of BIM and LCA } \\
\text { integration based on literature review. } \\
\text { Developed a viable method organize building } \\
\text { information based on the BIM model to estimate the } \\
\text { impact on the environment and energy consumption. } \\
\text { The study also proposed the templates and plug-ins } \\
\text { including the integration of automation for data. }\end{array}$ & $\begin{array}{l}\text { - LCA applied at the early stage which allows } \\
\text { the designers to select materials, transport } \\
\text { distances, etc. with different construction } \\
\text { techniques, etc. } \\
\text { - Quick and reliable environmental performance } \\
\text { of buildings. } \\
\text { - Challenging in terms of interoperability. }\end{array}$ \\
\hline $\begin{array}{c}\text { Santos et al. } \\
{[42]}\end{array}$ & $\begin{array}{l}\text { - Identified the required information for LCA and } \\
\text { incorporated it into the BIM model. } \\
\text { Analyzed energy and environmental impacts of two } \\
\text { different buildings comparatively to understand how } \\
\text { designers' choices affect building performance. } \\
\text { Identified the most influential factors of } \\
\text { environmental impacts of buildings by analyzing the } \\
\text { use of different materials and the phases. }\end{array}$ & $\begin{array}{l}\text { Wood-based solutions can potentially suppress } \\
\text { most of the environmental impacts due to } \\
\text { lower primary energy demand and higher } \\
\text { end-of-life potential use. } \\
\text { The concrete structure was associated with very } \\
\text { high impacts compared with other materials. }\end{array}$ \\
\hline $\begin{array}{l}\text { Martin et al. } \\
{[22]}\end{array}$ & $\begin{array}{l}\text { Established a link between LCA and BIM. } \\
\text { To support decision-making, the study proposed } \\
\text { workflow of using conceptual BIM models and } \\
\text { visual scripts by considering several construction } \\
\text { options. } \\
\text { Identify design specific hotspots for different choices } \\
\text { based on the assessed environmental impacts. }\end{array}$ & $\begin{array}{l}\text { An integration of LCA into BIM is possible } \\
\text { when a unified standard is used in both LCI } \\
\text { data and BIM aided bill of quantities. } \\
\text { It helps to identify the hotspots of } \\
\text { design-specific features. }\end{array}$ \\
\hline $\begin{array}{c}\text { Schultz et al. } \\
{[12]}\end{array}$ & $\begin{array}{l}\text { - Compared Tally Plugin and the Athena Impact } \\
\text { Estimator based on the user inputs, outputs, LCI } \\
\text { databases, interfaces and the discrepancies between } \\
\text { them, inputs and outputs, etc. } \\
\text { Developed a guideline for LCA of the entire building } \\
\text { based on BIM. }\end{array}$ & $\begin{array}{l}\text { - Integrated BIM-based LCA can be effectively } \\
\text { used in whole-building assessment at the } \\
\text { design process, and in identifying the potential } \\
\text { improvement measures for long term } \\
\text { sustainability of the buildings. } \\
\text { However, it needs expertise to perform } \\
\text { BIM-based LCA of building, otherwise the } \\
\text { tools including specific data input may be } \\
\text { biased which affects LCA outcomes. }\end{array}$ \\
\hline
\end{tabular}

- Carbon emission of building was evaluated.

- The operational stage emitted higher amount than other stages, whereas carbon sequestration by

Peng [11] vegetation was minimal.

- A sensitivity analysis by changing parameters for

- By optimizing the operation phase of the building, the government can minimize carbon emissions. identifying the influential factors on building performance was conducted.

- $\quad$ Proposed an integrated automated model that links several tools including BIM, LCA, energy and

Jalaei et al. lighting simulation tools for using in the green [21] building certification systems.

- $\quad$ Plug-in on BIM tool capable of calculating embodied energy of building components.
- Reviewed the current implications of BIM for sustainability assessment.

- Investigated the potential BIM standards such as IFC,

Ramaji et al. [44] application challenges.

- Provided an LCA analysis approach based on IFC/LOD for analyzing the embodied energy of buildings.
- Can assist in selecting different materials and components, based on the comparative analysis.

- It can modify the building components at the conceptual design stage.

- Conducted sustainability evaluation at an early stage of the project, where possible changes can be possible with a lower cost, but imposes a much higher impact on sustainability.

- $\quad$ Proposed three levels of IFC-based LCA analysis such as generic, system and component levels. 
Table 1. Cont

\begin{tabular}{|c|c|c|}
\hline Authors & Study Highlights & Implications \\
\hline $\begin{array}{l}\text { Wang et al. } \\
\quad[45]\end{array}$ & $\begin{array}{l}\text { - Computationally integrates BIM, LCA, energy } \\
\text { including the optimization software for evaluating } \\
\text { the environmental impacts of buildings. } \\
\text { The multi-objective genetic algorithm implements a } \\
\text { Pareto optimal solution to minimize costs and } \\
\text { environmental impact. }\end{array}$ & $\begin{array}{l}\text { - Complicated when large/multi-storied } \\
\text { buildings with different other variables such as } \\
\text { occupant behavior, waste management } \\
\text { systems, recycling, etc., are integrated into the } \\
\text { optimization model. }\end{array}$ \\
\hline $\begin{array}{l}\text { Basbagill et al. } \\
\text { [41] }\end{array}$ & $\begin{array}{l}\text { A BIM-based LCA was proposed to facilitate the } \\
\text { decision process for selecting building components } \\
\text { and materials based on their relative environmental } \\
\text { impacts. } \\
\text { - Sensitivity analysis was conducted for a range of } \\
\text { building characteristics and design parameters. }\end{array}$ & $\begin{array}{l}\text { Environmental impacts for service equipment } \\
\text { are relatively small, but significant for cladding } \\
\text { material and their thickness choices, regardless } \\
\text { of building designs. }\end{array}$ \\
\hline Lee et al. [43] & $\begin{array}{l}\text { Developed a template for evaluating the } \\
\text { environmental impact of building based on a BIM } \\
\text { design tool. }\end{array}$ & \\
\hline $\begin{array}{l}\text { Soust- } \\
\text { Verdaguer } \\
\text { et al. [40] }\end{array}$ & $\begin{array}{l}\text { - To identify the simplification strategies and to } \\
\text { promote further developments in building LCA, an } \\
\text { extensive review was conducted. } \\
\text { Examined system boundaries, sources of data/ } \\
\text { inventory data, life cycle phases included and impact } \\
\text { considerations. }\end{array}$ & $\begin{array}{l}\text { - Global warming potential was the most } \\
\text { considered impact category. } \\
\text { Simplified methods will affect the definition of } \\
\text { the physical model, life cycle scenario and the } \\
\text { communication of results. }\end{array}$ \\
\hline $\begin{array}{l}\text { Schwartz } \\
\text { et al. [26] }\end{array}$ & $\begin{array}{l}\text { An embedded framework was proposed by } \\
\text { integrating BIM and LCAs to support the } \\
\text { decision-making process for achieving } \\
\text { environmental responsible designs. }\end{array}$ & $\begin{array}{l}\text { - Semantic representation of BIM models can be } \\
\text { utilized for material EPD specifications. } \\
\text { The ifc XML-BIM integration should be } \\
\text { adopted, as importing the IFC model into BIM } \\
\text { can result in some data loss. } \\
\text { The enrichment of a BIM model with EPD data, } \\
\text { the integration of other domains such as cost } \\
\text { data within BIM using ontology and semantic } \\
\text { web should also be explored. }\end{array}$ \\
\hline $\begin{array}{l}\text { Kulahcioglu } \\
\text { et al. [46] }\end{array}$ & $\begin{array}{l}\text { - Proposed an IFC-based BIM using a built-in material } \\
\text { inventory. }\end{array}$ & $\begin{array}{l}\text { - Availability including the quality of LCI data } \\
\text { greatly affect the accuracy of LCA results. } \\
\text { The study approach, however, failed to address } \\
\text { the key issues associated with LCI obtained } \\
\text { from the user inputs (For example, the built-in } \\
\text { inventory was fixed and could not be altered). }\end{array}$ \\
\hline $\begin{array}{l}\text { Mao et al. } \\
\text { [47] }\end{array}$ & $\begin{array}{l}\text { Presented a new decision-making tool which } \\
\text { integrates BIM and carbon emission tool to calculate } \\
\text { the embodied emission of building materials, and to } \\
\text { optimize the selection of low carbon materials. }\end{array}$ & $\begin{array}{l}\text { - Limited indicators only. } \\
\text { Not considered the recycling materials / waste } \\
\text { management towards CE adoption. }\end{array}$ \\
\hline $\begin{array}{l}\text { Ajayi et al. } \\
\text { [24] }\end{array}$ & $\begin{array}{l}\text { - Assess the impact of buildings on the life cycle from } \\
\text { the perspective of fossil fuels and renewable energy. } \\
\text { By comparing health and environmental impact to } \\
\text { assess the health of eco-buildings. }\end{array}$ & $\begin{array}{l}\text { - Use renewable energy in buildings showed } \\
\text { healthy environmental performance than the } \\
\text { energy from fossil fuels, irrespective of } \\
\text { materials used. } \\
\text { The embodied impact must be carefully noted } \\
\text { with more and more efficient building } \\
\text { achieving during the operational phase. } \\
\text { Layout a methodological framework for } \\
\text { practitioners in selecting the materials based on } \\
\text { BIM model and evaluating the lifecycle impacts } \\
\text { of building at design stage. }\end{array}$ \\
\hline
\end{tabular}


Table 2. Different considerations in the existing BIM-LCA studies.

\begin{tabular}{|c|c|c|c|c|c|c|c|c|}
\hline Authors & Location & $\begin{array}{l}\text { Selected } \\
\text { Materials }\end{array}$ & Methods & Tools & $\begin{array}{l}\text { Evaluation } \\
\text { Indicators }\end{array}$ & $\begin{array}{c}\text { Function } \\
\text { Unit }\end{array}$ & $\begin{array}{c}\text { LCA } \\
\text { Phase }\end{array}$ & $\begin{array}{l}\text { Significant } \\
\text { Findings }\end{array}$ \\
\hline $\begin{array}{l}\text { Santos } \\
\text { et al. [42] }\end{array}$ & Portugal & $\begin{array}{l}\text { Concrete, } \\
\text { masonry, } \\
\text { metals, } \\
\text { thermal and } \\
\text { moisture } \\
\text { protection, } \\
\text { openings } \\
\text { and glazing, } \\
\text { finishes }\end{array}$ & LCA plug-in & $\begin{array}{l}\text { Revit; Tally; } \\
\text { TRACI } 2.1 .\end{array}$ & $\begin{array}{c}\text { AP, EP, GWP, } \\
\text { ODP, FP } \\
\text { Primary } \\
\text { Energy } \\
\text { Demand }\end{array}$ & $\begin{array}{l}\text { Residential } \\
\text { buildings }\end{array}$ & $\begin{array}{l}\text { Cradle-to- } \\
\text { grave }\end{array}$ & $\begin{array}{l}\text { Wood-based } \\
\text { solutions leading } \\
\text { to greater } \\
\text { environmental } \\
\text { benefits, and the } \\
\text { worst for } \\
\text { concrete } \\
\text { envelope }\end{array}$ \\
\hline $\begin{array}{l}\text { Martin } \\
\text { et al. [22] }\end{array}$ & Switzerland & $\begin{array}{c}\text { External } \\
\text { walls; } \\
\text { external } \\
\text { layers } \\
\text { including } \\
\text { insulation; } \\
\text { structural } \\
\text { layer(s); and } \\
\text { interior } \\
\text { finishing }\end{array}$ & $\begin{array}{c}\text { Dynamo } \\
\text { script in } \\
\text { Microsoft } \\
\text { Excel and the } \\
\text { BIM model } \\
\text { in Autodesk } \\
\text { Revit }\end{array}$ & $\begin{array}{c}\text { Revit; } \\
\text { Dynamo }\end{array}$ & GWP & $\begin{array}{l}\text { Building } \\
\text { elements: } \\
\text { foundation } \\
\text { slab, external } \\
\text { walls, floor } \\
\text { and roof } \\
\text { elements, } \\
\text { windows } \\
\text { and partition } \\
\text { walls. }\end{array}$ & $\begin{array}{l}\text { Cradle-to- } \\
\text { gate }\end{array}$ & $\begin{array}{l}\text { The element } \\
\text { class of floors } \\
\text { and partition } \\
\text { walls have a } \\
\text { higher deviation } \\
\text { in impact than } \\
\text { foundations, } \\
\text { roofs, external } \\
\text { walls and } \\
\text { windows }\end{array}$ \\
\hline $\begin{array}{l}\text { Basbagill } \\
\text { et al. [41] }\end{array}$ & USA & $\begin{array}{l}\text { Included } 95 \\
\text { sub- } \\
\text { components }\end{array}$ & $\begin{array}{l}\text { Converting } \\
\text { building } \\
\text { component } \\
\text { material } \\
\text { quantities } \\
\text { into } \\
\text { embodied } \\
\text { impacts }\end{array}$ & $\begin{array}{l}\text { Dprofiler; } \\
\text { eQUEST; } \\
\text { SimaPro }\end{array}$ & $\begin{array}{l}\text { Material } \\
\text { densities; } \\
\text { Embodied } \\
\mathrm{CO}_{2} \mathrm{e} \text {, or the } \\
\text { amount of } \\
\text { carbon } \\
\text { dioxide } \\
\text { equivalents }\end{array}$ & $\begin{array}{l}\text { Residential } \\
\text { building }\end{array}$ & $\begin{array}{l}\text { Cradle-to- } \\
\text { cradle }\end{array}$ & $\begin{array}{c}\text { Service } \\
\text { equipment } \\
\text { contributed less } \\
\text { impact, whereas } \\
\text { significantly } \\
\text { higher for } \\
\text { cladding } \\
\text { material and the } \\
\text { choice of } \\
\text { thickness }\end{array}$ \\
\hline $\begin{array}{l}\text { Lee et al. } \\
\text { [43] }\end{array}$ & South Korea & $\begin{array}{l}\text { Ready- } \\
\text { mixed } \\
\text { concrete, } \\
\text { glass, } \\
\text { concrete } \\
\text { block, } \\
\text { insulation } \\
\text { material and } \\
\text { gypsum } \\
\text { board }\end{array}$ & $\begin{array}{l}\text { Extracted the } \\
\text { quantity } \\
\text { takeoff; then } \\
\text { performed } \\
\text { an } \\
\text { evaluation }\end{array}$ & Revit & $\begin{array}{l}\text { GWP, ADP, } \\
\text { EP, ODP, PP }\end{array}$ & $\begin{array}{c}\text { Material } \\
\text { levels }\end{array}$ & $\begin{array}{l}\text { Cradle-to- } \\
\text { grave }\end{array}$ & $\begin{array}{c}\text { Table for } \\
\text { checking real } \\
\text { time } \\
\text { environmental } \\
\text { impacts }\end{array}$ \\
\hline $\begin{array}{l}\text { Schwartz } \\
\text { et al. [26] }\end{array}$ & UK & $\begin{array}{c}\text { Included } 35 \\
\text { different } \\
\text { object } \\
\text { properties }\end{array}$ & $\begin{array}{c}\text { Examined } \\
\text { the } \\
\text { application } \\
\text { of semantic } \\
\text { rules on BIM } \\
\text { models } \\
\text { through the } \\
\text { ifcXML } \\
\text { protocol by } \\
\text { using } \\
\text { Semantic } \\
\text { Web } \\
\text { applications }\end{array}$ & $\begin{array}{c}\text { Revit; Pellet } \\
1.5 .2\end{array}$ & $\begin{array}{c}\text { Embodied } \\
\mathrm{CO}_{2} \\
\text { emission }\end{array}$ & $\begin{array}{l}\text { Buildings } \\
\text { material }\end{array}$ & $\begin{array}{l}\text { Cradle-to- } \\
\text { site }\end{array}$ & $\begin{array}{l}\text { Framework for } \\
\text { representing, } \\
\text { managing and } \\
\text { deploying } \\
\text { building } \\
\text { information } \\
\text { instantly across } \\
\text { different data } \\
\text { sources was } \\
\text { presented by } \\
\text { using semantic } \\
\text { web technique }\end{array}$ \\
\hline $\begin{array}{c}\text { Mao et al. } \\
\text { [47] }\end{array}$ & China & $\begin{array}{c}\text { Concrete, } \\
\text { cement, } \\
\text { sand, steel, } \\
\text { glass, } \\
\text { aluminum, } \\
\text { ceramic } \\
\text { brick, brick, } \\
\text { gypsum } \\
\text { wallboard, } \\
\text { polyvinyl } \\
\text { chloride } \\
\text { (PVC) }\end{array}$ & $\begin{array}{l}\text { A BIM-GHG } \\
\text { plug-in }\end{array}$ & $\begin{array}{l}\text { Autodesk, } \\
\text { Bentley, } \\
\text { Nemetscheck; } \\
\text { Graphisoft; } \\
\text { BIM }\end{array}$ & $\begin{array}{l}\text { GHG } \\
\text { emission }\end{array}$ & $\begin{array}{l}\text { Building } \\
\text { materials }\end{array}$ & $\begin{array}{l}\text { Cradle-to- } \\
\text { gate }\end{array}$ & $\begin{array}{l}\text { Used to select } \\
\text { low carbon } \\
\text { building } \\
\text { materials in the } \\
\text { design stage for } \\
\text { cut down GHG } \\
\text { emissions from } \\
\text { every stage. }\end{array}$ \\
\hline $\begin{array}{c}\text { Wang et al. } \\
{[48]}\end{array}$ & USA & $\begin{array}{l}\text { Steel, mortar, } \\
\text { ready-mixed } \\
\text { concrete, } \\
\text { doors and } \\
\text { glass }\end{array}$ & $\begin{array}{l}\text { BIM Model; } \\
\text { Ecotect }\end{array}$ & $\begin{array}{l}\text { Ecotect and } \\
\text { Autodesk }\end{array}$ & $\begin{array}{l}\mathrm{CO}_{2} \\
\text { emission and } \\
\text { energy con- } \\
\text { sumption }\end{array}$ & $\begin{array}{l}\text { University } \\
\text { building }\end{array}$ & $\begin{array}{l}\text { Cradle-to- } \\
\text { grave }\end{array}$ & $\begin{array}{c}\text { Majority of } \\
\text { energy } \\
\text { consumption } \\
\text { was reported in } \\
\text { the operating } \\
\text { stage. }\end{array}$ \\
\hline
\end{tabular}


Table 2. Cont.

\begin{tabular}{|c|c|c|c|c|c|c|c|c|}
\hline Authors & Location & $\begin{array}{l}\text { Selected } \\
\text { Materials }\end{array}$ & Methods & Tools & $\begin{array}{l}\text { Evaluation } \\
\text { Indicators } 1\end{array}$ & $\begin{array}{c}\text { Function } \\
\text { Unit }\end{array}$ & $\begin{array}{c}\text { LCA } \\
\text { Phase }\end{array}$ & $\begin{array}{l}\text { Significant } \\
\text { Findings }\end{array}$ \\
\hline $\begin{array}{c}\text { Ajayi et al. } \\
{[24]}\end{array}$ & UK & $\begin{array}{c}\text { Timber, } \\
\text { brick/block, } \\
\text { steel, } \\
\text { insulated } \\
\text { concrete } \\
\text { foams }\end{array}$ & $\begin{array}{c}\text { BIM } \\
\text { incorporated } \\
\text { LCA }\end{array}$ & $\begin{array}{l}\text { Revit, Green } \\
\text { Building } \\
\text { Studio, } \\
\text { ATHENA } \\
\text { Impact } \\
\text { Estimator }\end{array}$ & $\begin{array}{l}\text { GWP and } \\
\text { health } \\
\text { impacts }\end{array}$ & $\begin{array}{l}\text { One- } \\
\text { storyschool } \\
\text { building }\end{array}$ & $\begin{array}{l}\text { Cradle-to- } \\
\text { grave }\end{array}$ & $\begin{array}{l}\text { In terms of } \\
\text { building } \\
\text { materials, both } \\
\text { environmental } \\
\text { and health } \\
\text { preference of } \\
\text { buildings } \\
\text { congruently } \\
\text { rangefrom } \\
\text { timber, } \\
\text { brick/block, } \\
\text { steel, to insulated } \\
\text { concrete foams, } \\
\text { in descending } \\
\text { order. }\end{array}$ \\
\hline $\begin{array}{l}\text { Julianna } \\
\text { et al. [49] }\end{array}$ & Brazil & $\begin{array}{c}\text { Brazilian } \\
\text { conventional } \\
\text { masonry } \\
\text { (clay brick), } \\
\text { concrete } \\
\text { block } \\
\text { masonry, } \\
\text { steel frame } \\
\text { and wood } \\
\text { frame. }\end{array}$ & $\begin{array}{c}\text { BIM } \\
\text { incorporated } \\
\text { LCA }\end{array}$ & $\begin{array}{l}\text { SimaPro 8; } \\
\text { ArchiCAD }\end{array}$ & $\begin{array}{c}\text { GHGs } \\
\text { emissions }\end{array}$ & $\begin{array}{l}\text { Four } \\
\text { different } \\
\text { types of } \\
\text { interior } \\
\text { walls. }\end{array}$ & $\begin{array}{l}\text { Cradle-to- } \\
\text { gate }\end{array}$ & $\begin{array}{l}\text { Concerning the } \\
\text { walls, the wood } \\
\text { frame system } \\
\text { was the most } \\
\text { sustainable and } \\
\text { most } \\
\text { economically } \\
\text { feasible option }\end{array}$ \\
\hline $\begin{array}{c}\text { Soust- } \\
\text { Verdaguer } \\
\text { et al. [50] }\end{array}$ & Uruguay & $\begin{array}{l}\text { bricks and } \\
\text { concrete } \\
\text { blocks }\end{array}$ & $\begin{array}{l}\text { BIM and } \\
\text { other tools }\end{array}$ & $\begin{array}{c}\text { ArchiCAD; } \\
\text { Excel; } \\
\text { Design- } \\
\text { Builder;Energy } \\
\text { Plus } \\
\text { Ecodesigner } \\
\text { STAR }\end{array}$ & $\begin{array}{l}\text { GWP, } \\
\text { freshwater } \\
\text { aquatic } \\
\text { ecotoxicity, } \\
\text { human } \\
\text { toxicity, ODP }\end{array}$ & $\begin{array}{l}\text { Single- } \\
\text { family } \\
\text { houses }\end{array}$ & $\begin{array}{l}\text { Cradle-to- } \\
\text { grave }\end{array}$ & $\begin{array}{l}\text { Aerated concrete } \\
\text { blocks have high } \\
\text { carbon emission } \\
\text { than the normal } \\
\text { concrete blocks }\end{array}$ \\
\hline $\begin{array}{l}\text { Cristiane } \\
\text { et al. [51] }\end{array}$ & Brazilian & $\begin{array}{c}\text { Concrete } \\
\text { blocks } \\
\text { masonry } \\
\text { original clay } \\
\text { tiles }\end{array}$ & $\begin{array}{l}\text { Visual pro- } \\
\text { gramming } \\
\text { routine }\end{array}$ & $\begin{array}{l}\text { GaBi; } \\
\text { Autodesk } \\
\text { Revit; } \\
\text { Microsoft } \\
\text { Excel }\end{array}$ & $\longrightarrow$ & $\begin{array}{l}1 \mathrm{~m}^{2} \text { of the } \\
\text { non- } \\
\text { structural } \\
\text { shell }\end{array}$ & $\begin{array}{l}\text { Cradle-to- } \\
\text { grave }\end{array}$ & $\begin{array}{l}\text { The concrete } \\
\text { blocks masonry } \\
\text { provides the best, } \\
\text { while the clay } \\
\text { tiles have a better } \\
\text { environmental } \\
\text { performance } \\
\text { than the fiber } \\
\text { cement option. }\end{array}$ \\
\hline $\begin{array}{c}\text { Yang et al. } \\
\text { [52] }\end{array}$ & $\begin{array}{l}\text { Chongqing, } \\
\text { China }\end{array}$ & $\begin{array}{l}\text { Cement, } \\
\text { pinewood, } \\
\text { hot rolled } \\
\text { steel bar, } \\
\text { steel plate, } \\
\text { steel bar, } \\
\text { concrete } \\
\text { brick; } \\
\text { aluminum } \\
\text { alloy, sheet } \\
\text { glass }\end{array}$ & $\begin{array}{l}\text { BIM-enabled } \\
\text { LCA method }\end{array}$ & $\begin{array}{c}\text { Autodesk } \\
\text { Revit 2015; } \\
\text { Design } \\
\text { builder }\end{array}$ & GWP & $\begin{array}{l}\text { Village } \\
\text { building }\end{array}$ & $\begin{array}{l}\text { Cradle-to- } \\
\text { grave }\end{array}$ & $\begin{array}{l}\text { Steel and } \\
\text { aluminum } \\
\text { contribute to } \\
\text { higher GHG } \\
\text { emissions than } \\
\text { concrete. }\end{array}$ \\
\hline
\end{tabular}

${ }^{1}$ AP, EP, GWP, ODP, FP, PP represent acidification potential, eutrophication potential, global warming potential, ozone depletion potential, formation potential, photochemical ozone creation potential, respectively.

\subsection{Why the BIM Integrated LCA Is Important}

As the integrated BIM-LCA can be considered as a powerful approach to perform environmental evaluation of buildings at the design stage, an increasing number of applications is observed recently $[40,53,54]$. BIM-based sustainable design can greatly change traditional design, as the BIM model can support more sustainable design and redesign based on the environmental performance, and also facilitate rigorous management of environmental impacts of construction facilities [4].

Moreover, studies demonstrated the necessity for integration of LCA and BIM towards sustainable construction projects $[55,56]$. Such integration and assessment should be at the early stage that allows quick modeling and obtained reliable results $[7,57]$. The primary data used for LCA can be gathered from a case-specific BIM design, not only increases the efficiency of the previously boring LCA with an accuracy of the assessment, but also 
decrease the temporal and spatial complexity for sustainable building design. From this integrated system, the designers will be convenient from the LCA results towards designing a sustainable building by changing the design, materials, components or the process that provides the opportunity of pre-control enhancement for sustainable construction [58].

\subsection{How to Achieve Sustainability through "BIM Integrated LCA"?}

For achieving/enhancing sustainability, BIM can be integrated with LCA at three levels: (i) the first level is the use of BIM to quantify materials and architectural elements for LCI data, (ii) the second level, the integration of environmental information to BIM as a design tool and (iii) the last level is to develop an automated process based on LCI data and software [7]. Some studies proposed a two-approach of BIM-based LCA. The first approach is the information during its entire lifecycle can be obtained directly from the BIM model, while the second method is to establish an automatic and effective connection between the BIM model and the environmental information, and mainly material-oriented, may be seen as a way of including environmental criteria on the same level as other features in the early design phases [3].

In most of cases, the information from BIM platform for every material quantity were collected and manually organized for LCA application [7]. However, a few studies highlighted that BIM integrated LCA can be divided into (i) operational stage (building phase) such as construction, building in-use and demolition, and (ii) pre-operational stage (embodied phase) that involves the building materials production and collection [59]. In the operational phase, BIM can be used to optimize energy analysis based on the building geometry at the beginning of modeling. However, some gaps have can be observed between predicted energy performance. For example, the use of historical environmental data to predict future occupant's behavior is generally inaccurate. In addition, geometric features are easily lost during data exchange or model conversion. In the pre-operational stage, calculating the embodied energy in architectural elements requires two sets: the size/number of elements and their associated LCI databases (for upstream data). The total energy or impact of a building element or material could be calculated by multiplying the size/number of elements or material by the corresponding factors from LCI database. Detailed information of the BIM model enhances the accuracy of environmental evaluation, while the automated quantity take-off process significantly reduces the required analysis time [44].

A typical BIM and LCA integration at the early-stage design of a construction project is given in Figure 4 (adapted by Jalaei [21]). It was separated into 4 aspects including material selection, building model, sustainable analysis and evaluation. Since the framework aimed at materials selection, energy simulation, passive design and LCA were set in the same level of sustainable design, because energy simulation tools always utilized the related passive design of the building and its materials. In general, the components analyzed by energy simulation from BIM tools had their specifications as close as possible to the ones used in the real design. Through adjusting the components parameters, designers can get the level of energy consumption to compare the effects of components and analyze energy-savings of case building and optimize the components or building material. The results can be as a very important reference for passive design in Green Building Standard in the popular evaluation system in the world, for example calculating the accumulated Leadership in Energy \& Environmental Design (LEED) points that can potentially be earned during the conceptual design stage. For the analysis of LCA, every component forms building model quantity take-offs, such as the floor, walls, the roof and windows have their associated LEED information linked to the families of the BIM tool and they are already defined in the database of LCA tools/plug-in (SimaPro, EcoScorecard), which can achieve the environment impact to guide the selecting of material. Based on the results, the evaluation of a building or its materials can be a comprehensive assessment in sustainability to assist designers in comparing and analyzing each design family and its associated components that is selected during the conceptual design. 


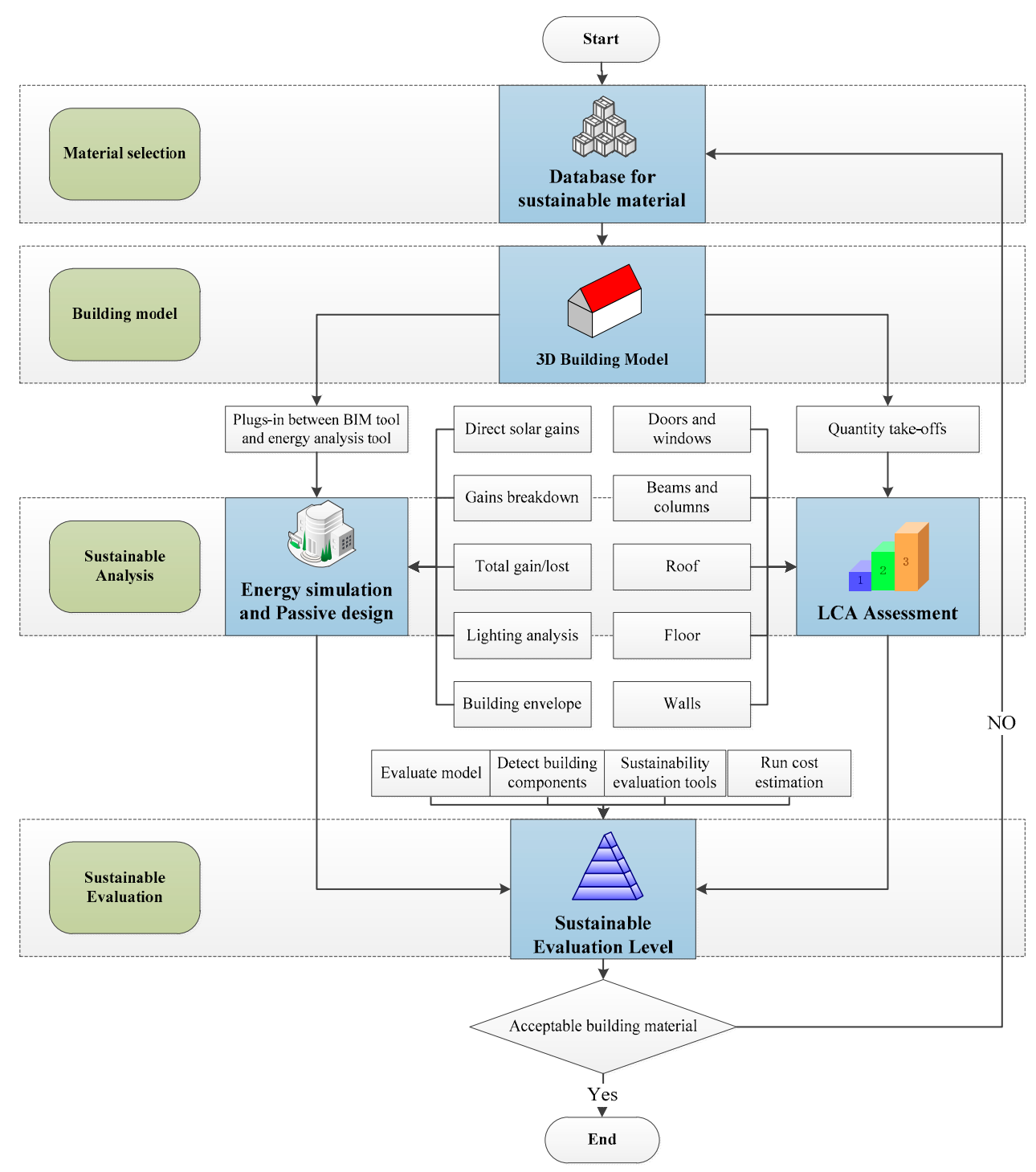

Figure 4. Framework of BIM integrated LCA for materials selection.

\subsection{LCA and BIM for Construction Sustainability: Challenges}

Although the BIM and LCA integration platform can significantly shorten time and add the applications for evaluating environmental performance, several challenges still can be highlighted, such as: (i) methodological challenge, (ii) interoperability, (iii) insufficient case studies and data in BIM to provide the LCA applications, (iv) methodological limitations of scenario verification of different materials and (v) the level of required model and information for LCA [22]. The key issue is still these two disciplines remain separated with virtually little overlap in tools, terminologies and data structures [11]. Thus, BIM integrated LCA has a certain methodological challenge. The BIM application only stays in taking-off quantities and measures the size of the element or simply interacting with the LCA (e.g., manual input) [44]. As a result, construction evaluation will still be complex and time-consuming. So far, most BIM-based LCA studies have focused on one-time post assessment instead of iterative assessment during building design for sustainable decision [22].

In addition, the typologies used in the LCA studies were not readily available in the Revit default models. Based on the analyzed case studies, the data available in BIM databases are not sufficient to provide a comprehensive LCA model [7]. Although the plugin system allows the users to easily export the materials and component take-off quantity and then link them to other tools including LCA [21], this may not recognize the object's 
information, meaning the required information should be manually added after designing the architecture including the type of solutions in the Revit [42]. The assumptions-based simpler BIM design may lead to discrepant results from the plug-in and LCA analysis [51]. Peng et al. highlighted BIM cannot develop enough data in the early design phase for the comprehensive LCA, as the current purpose of these two is vastly different [11]. In addition, comprehensive methodological details and frameworks for integrating BIM and LCA is still lacking that can be used in decision-making [60]. Apart from what is highlighted above, some other limitations are demonstrated in the prevailing studies such as: (i) mostly focused on a few specific building components instead of the entire building, (ii) the annual actual electricity and water consumption are derived from similar buildings (e.g., using statistics), but greatly varied according to temporal and spatial contexts, (iii) since the complex characteristics of the construction and renovation process, most need to be super-simplified or eliminated and (iv) limited automation and interoperability by using formats like IFC and gbXML cannot significantly reduce manual time [52].

\subsection{Tools or Plug-In for Integrating BIM into LCA}

Through utilizing different tools and/or plug-in systems, it is possible to output the materials' quantity and connect them to LCA [21]. Several software have been used to assess the environmental impact of buildings at the early stage. For example, the Athena EcoCalculator was used for environmental impact estimation with minimal inputs [61]. However, when the design alternates, these tools cannot analyze the environmental impact of different building components. Moreover, there is no integration with BIM tools which further decreases their effectiveness during the early design stage. DProfiler was used as the selected BIM software in several studies [62-64] which provides the information of materials and energy uses with minimal building design inputs, but it has limitations in terms of the range of geometric forms that it created. In addition, BIM-based program can be outputted to eQUEST for analyzing the energy performance of a building [65], but requires long run times, and only analyzed the energy performance [41]. SimaPro is a leading LCA software that contains thousands of databases for many different building materials, products, transportation and processes $[25,66]$. However, SimaPro is a standalone LCA software for diverse disciplines, and no attempt is found so far to integrate into BIM for computation of environmental impacts. Athena EcoCalculator is used for evaluating building footprints, but lacks to considered building component thickness instead of only building gross floor area. CostLab is the online facility cost tool used to estimate and forecast operational, maintenance, repair costs, etc. Since the program is not an LCA tool, the results output the predicted cost of building components, instead of the specific environmental impact [41].

Other tools like IMPACT, were used to evaluate environmental impacts based on BIM in the standard IFC and gbXML formats, developed by the UK Building Research Establishment. However, there is a lack of inter-data compatibility, and unsupported data format (especially for LCA tools), which requires additional processing time [43]. Schultz et al. highlighted tools that integrate BIM into LCA such as Revit's Tally plugin and LCA Design, developed by the Cooperative Research Centre for Construction Innovation in Australia. This tool can calculate environmental impacts at the early design stage with 3D drawing files through connected with the Australian LCI database [12]. However, the challenges of some tools and plug-ins about the integration of BIM-LCA have been underlined in some studies. For example, LCA plug-in did not recognize the elements information such as the chosen materials correctly, requiring manually processing and adding into the LCA tools [42].

\section{Circular Economy and the Building Industry}

\subsection{Concept and Implications}

The principle of circular economy (CE) is a sub-branch industrial ecology, has recently gained enormous attention in all sectors over the globe. $\mathrm{CE}$ is defined "as a regenerative 
system in which resource input and waste, emission, and energy leakage are minimized by slowing, closing, and narrowing material and energy loops" $[28,29]$. Due to increasing concern over resources use, both CE and sustainability are interconnected concepts, becoming popular among the policymakers, academia and industry, and thus, numerous efforts are devoted to establishing the conceptual interrelationship between CE and sustainability $[28,29]$.

It is assumed that three major potential benefits including economic, social and environmental benefits can be achieved for implementing the CE model [28,29]. It can highly improve the local and regional competitiveness through increasing effectiveness of the resource allocation, utilization and productivity which can lead to greater economic stability as well as resource security [67]. The model can reduce the negative environment impacts through redesigning of industrial structure or production system in an ecological way and saving the primary resources [68]. Moreover, CE model can increase the additional employment opportunities, economic growth, etc. [69]. Based on establishing the interrelationship between ecological systems and economic growth, $\mathrm{CE}$ framework especially highlights the environmental sustainability of a system [70].

\section{2. $C E$ and Buildings}

When CE principle is integrated to buildings, it can help in achieving the several objectives: (i) improvement of materials and energy efficiency through sourcing of sustainable materials and enhancing the collaborative benefits between the associated industries [29,30], (ii) maximize recycling and reuse of materials and components, and significantly reduce waste generation [31], (iii) avoid waste disposal problems and save landfill space [32]. Furthermore, moving towards a CE model provides an opportunity to reduce the use of primary materials, ensure material efficiency and reduce associated environmental impacts.

The main goal of $\mathrm{CE}$ is to limit new material extraction while retaining the economics of the extracted materials for a long time to reduce the residual waste. This is extremely important for the building industry, which consumes a huge number of natural resources globally and generates a considerable volume of waste annually. For achieving long run sustainability, the use of the CE concepts may be the key strategy for in-depth assessment, as it already includes environmental, social, economic, business and policy aspects into the CE framework [70,71]. According to the UK Green Building Council, about 50\% of greenhouse gas emission was contributed by the construction industry in the country [72], and about $40 \%$ of waste generation was by the industry in the EU. Thus, the EU regards the $\mathrm{CE}$ package as one of the five priority industries in the construction industry [73].

However, the implementation of CE into the buildings is not straight forward, as buildings are unique entities and are often the results of one-off projects. Since each component used has a natural life cycle and all of them are connected in space and time, this feature increases its natural complexity. Furthermore, their service life and renovation during use phase will make an increasing uncertainty in future alternatives. [71]. End-of-life activities such as demolition are responsible for more than $50 \%$ of the entire waste generated by the construction industry. The design of material circularity is challenging issue. Thus, new technologies and design approaches are required with a view of the challenge of materials circularity for adopting $\mathrm{CE}$ approach, particularly from the traditional 'takemake-dispose' system to a circular perspective on material reuse.

However, CE in the building industry is still in the early stage of development, although a few frameworks including the guidelines were proposed. For example, industrial symbiosis with $\mathrm{CE}$ for buildings sustainability for collaboration and performance evaluation [30], integration of CE and eco-innovations [74] and proposed a collaboration tool for $\mathrm{CE}$ in the building [73]. Based on the online survey in the UK, Adams et al. [35] highlighted several challenges, such as lack of motivation (particularly to design for end-of-life of building components), lower value for recovered products, fragmented supply chain and lack of clear financial issue, lower priority for end-of-life during design, limited interest, awareness and knowledge, etc. for enhancing the level of awareness of $\mathrm{CE}$ and the con- 
struction industry. In addition, CE system boundary particularly for buildings, governance and management issues, scope of material and energy flows, etc. are remarkable challenges that were highlighted by Korhonen et al. [75].

$\mathrm{CE}$ in the construction including building sectors should consider the mentioned challenges, particularly appropriately selecting the system boundary by using the suitably integrated tools during the early stages of design [76]. However, such evaluation tools are still lacking that can be used in CE of building. The existing CE studies have focused on specific products or elements rather than the whole building [77], and therefore the complexities associated with buildings are yet to be addressed. $\mathrm{CE}$ indicators shall be based on LCA, MFA and material flow cost accounting [78]. Even with the material or component level, it is still unclear how the loss of materials in terms of quantity/quality due to different uses (e.g., circular uses) can handle in LCA when CE is considered [79]. Furthermore, challenges such as lack or little consideration of the economic aspects, the lack of a holistic approach across the supply chain, linear and traditional thinking, the low value of end-of-life construction products, etc., although there are few CE applications which were mainly focused on material selection and design considerations [35].

\subsection{CE, LCA and BIM for Sustainable Construction}

According to Sassanelli et al. [80], the most common methodology on CE regulations is LCA. CE has been spread by the LCA principles such as the cradle-to-cradle (C2C) system boundary [81]. Several studies highlighted that the adoption of LCA can achieve further to the understanding of the importance to recognize $\mathrm{CE}$ potentials (i.e., reuse and recycling) in buildings. $\mathrm{CE}$ approach could involve designing a product that can be reused as a whole as a modular system or recycled at the material level at the end of a specific life cycle [82]. As a result, the cradle-to-grave may not be fully suitable for the CE setting due to its consideration [83]. Few studied demonstrated that $C E$ requires closing material cycle loops through the upcycling rather than downcycling, increasing the responsibility of producers.

Some studies highlighted the importance of LCA in the CE agenda. For example, Pomponi et al. [71] suggested that LCA and material flow analysis (MFA) can be integrated for the effective implementation of CE, whereas BIM can be used as a supplementary design tool. As mentioned earlier, BIM reflected great benefits in engaging in the wholebuilding life cycle, guiding stakeholders to work together using decision tools to achieve the information interaction and managing interdependencies between the material and its environmental impact in the building process. The current tools are very difficult to assess the salvage value (i.e., reusing and recycling) of architectural elements and materials throughout the different life cycles [29]. Even numerous studies have focused on the BIM application for project design, but only a limited study has focused on refurbishment and demolition projects [53].

Due to accumulation of lifecycle information of a building, BIM can be effectively used for designing the building by considering the CE approach [84]. Thus, using BIM at the early stage can help recognize the material flow [29], where LCA can be adopted by considering the material flow of a building in all phases base on the cradle-to-cradle system boundary $[31,36,37]$. Construction and demolition waste management $[32,85,86]$, deconstruction [87] and salvage performance of structural components [29] were mostly focused on the adoption of CE of a building. Thus, cradle-to-cradle system boundary should be considered when LCA is conducted with focusing CE in the building [32]. Sanchez et al. [87] proposed a semi-automated selective deconstruction programming method for buildings using BIM to enhance the deconstruction planning for reusing the materials. In order to estimate the salvage performance of building elements at the early stage, Lukman et al. [29] developed a whole-life performance estimator based on BIM. In addition, Eberhardt et al. [83] conducted a comparative analysis of linear design versus a prospective circular design for recycling and reusing three common building components including concrete columns, window and roof. Although not comprehensive, the above- 
mentioned cases demonstrated the ways of BIM aided CE adoption and LCA application for building evaluation.

\section{Challenges and Prospective Framework for BIM-LCA Design for Sustainable Building with Evolving CE Principle}

It can be demonstrated that CE adoption in buildings through BIM-based LCA study is still very limited. Several major results are highlighted: (i) CE principle was often used in the recycling materials and secondary materials after the operating phase of a building and its components; (ii) recent LCA and BIM were not provided a whole-life assessment, especially in reusability and recyclability of the materials and refurbishment and demolition process of a building; (iii) CE adoption in building LCA is very thin; and (iv) the concept of $\mathrm{CE}$ needs to be implemented more deeply in the using of BIM integrated LCA to achieve a building sustainable. Therefore, this study highlighted some major drawbacks with their potential solutions for integrating CE into BIM-based LCA of buildings, which are included:

1. The way of CE integration into BIM-based LCA for comprehensive design and evaluation is merely considered into the existing studies: For addressing the first challenge, the modular design for different parts/elements can be integrated into the building and then can be dissembled after its service life. In this design, BIM can be a central tool for design, where LCA can be used for evaluating the environmental performance for the different uses after necessary repairment of the elements (based on different life cycles, from one building to another one). Based on the major issues in technical, informational, functional and organizational issues of the integration process, some research reveals the need to (i) create a synchronized LCA methodology; (ii) develop information databases that ontologically and semantically conform to the BIM environment; (iii) create a flawless and automated exchange of information between BIM and LCA tools. A more reliable integration of BIM and LCA would foster the optimization of the environmental performance of buildings [88].

2. Design challenge with BIM: For addressing the second challenge, the BIM platform should run through the design for the decision and quick adjustment of the building program. Nevertheless, a large number of design works exist with a low efficiency and heavy physical work. Literatures pointed out that the simpler strategy would be to add environmental data within the BIM model to facilitate the interaction between design and environmental performance assessment [89]. Future technology needs as intelligent as possible to improve the level of automation and visualization, for example, by implementing artificial intelligence opportunities and machine learning which can be used as a way to address decision-making during the building process in the future, such as smart mapping of components, elements, sub-elements and materials [90]. Through better managing of the design unit of BIM, a designer can achieve much faster work and save more time and vigor to consider the sustainability of building with CE and LCA concepts. Recent research explored more and more domains by integrated other dimensions (e.g., schedule management, facilities management) in BIM models in order to achieve more comprehensive analysis [91].

3. The building elements and products have a poor efficiency and low value to recover, which make construction as the most emission-intensive industry. Recently, an inconsistent price result in the pros and cons of the product at the market and a lack of business mechanisms influence the distribution of building materials. A lot of companies producing building components lack interest, awareness and knowledge in the manufacturing process and focus mainly on short-lived manufactured products. At the beginning of the manufacturing products, companies should limit new material extraction and residual waste reduction and improve the recycling rate after end-of-life analyzing the environmental impact of LCA tools. On the other hand, it is very important for companies to shift the concept from linear supply to circular supply chain as it exhibits lower carbon emissions. In the distribution of products, a database of sale 
and application as a whole and better management reflect a necessary strategy for a company. Using BIM technology can fulfill better management of product flow in all stages; further, this strategy can assist to build a much more reasonable financial mechanism.

4. Public understanding and acceptance. For addressing the fourth challenge, products and designs should have a label of CE or sustainable. Using LCA to assess the influencing of environment and mark if it can be recycling and reusing; under these circumstances, the public will gradually develop a good habit of sustainability.

5. Potential human health risk: Along with multiple environmental impact indicators, the human health risk should subjoin to the database of building products and components. Latest research indicated that the high health risk comes from the manufacturing and operation phases of building materials, particularly the passive contribution to acidification potential, global warming potential, ozone depletion potential and wasting of renewable energy [60]. BIM technology supplies a great and convenient method to build the database, which can be easily integrated with LCA in the concept of CE (for circular products).

6. Government support: CE application is still not very fast in the building industry due to the lack of incentive to design for end-of-life of products, and the lack of consideration for end-of-life during building design let building practitioners do not see the benefits such as economy and cost savings. The government should specify more incentives for new technology companies associated with circular products.

7. Construction industry adoption: The developer should decide the future construction direction of a new building and further cooperate with the government to understand the future policy. Building a BIM platform to fulfill better management to the design and construction should establish a full process responsibility system through CE based on BIM integrated LCA. In order to obtain the best solution building databases for the quantification of building materials, it should be added with information at various levels of detail in the BIM platform to allow a quick and agile adoption [92].

8. Circular value chain: The value chain including manufacturing, allocation, etc. are seldom involved in CE currently [93]. Thus, the existing system for the linear supply chain is needed to re-think the circular way of supply by discussing the way of remanufacturing, distribution and sales, to promote the customers. The promotion of material reusability (e.g., by the adoption of circularity index and public incentives) can be recommended to expand the application of BIM in the sustainable construction [94]. More in-depth research on this aspect is necessary, as CE can affect the sustainability, business model and innovation systems $[28,75,95]$.

9. The role of governments (i.e., policy), the role of matter (e.g., design, technology, materials) and the role of individuals (i.e., behavioral), were not explicitly considered into the existing studies: The policy can play an anchor rule in promoting CE into the building, if there is regulation (and incentives) to use recycle elements/materials into new/reimbursed buildings. Similarly, designers should emphasize to promote the recycled materials/elements into the design, and LCA can be used to promote the customers to accept the designs. Based on the highlighted challenges and opportunities, an integrated theoretical perspective framework for BIM-LCA design for sustainable building evolving $\mathrm{CE}$ is given in Figure 5. 


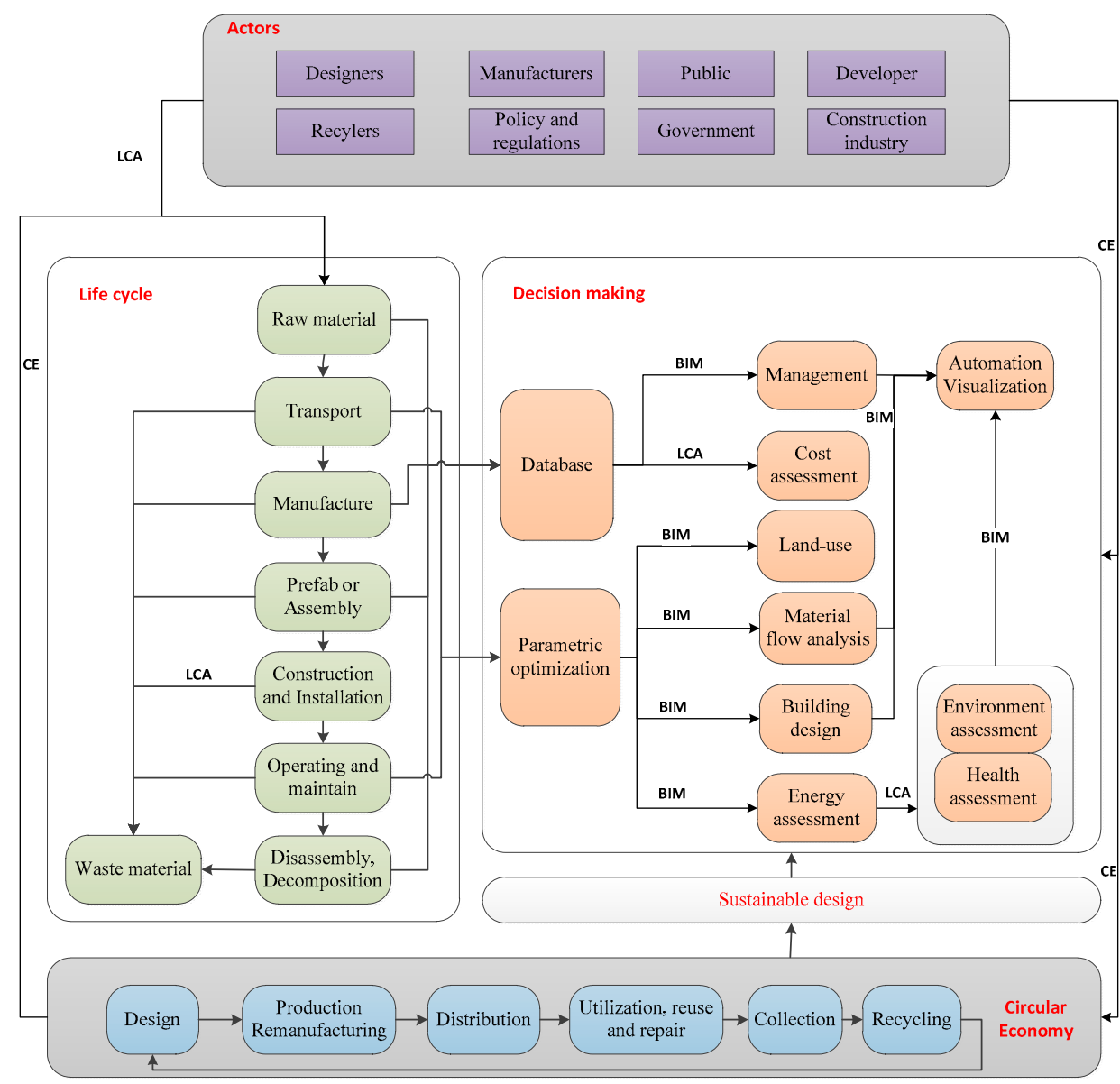

Figure 5. Integrated prospective framework for BIM-LCA design evolving circular economy (CE).

\section{Conclusions}

This study has provided critical insights into the BIM integrated LCA study of buildings and the possible integration of $\mathrm{CE}$ for promoting sustainability by critically analyzing the latest literature. The study concluded that scientific contributions in BIM, LCA and building had significantly increased in the past years due to growing attention on resource efficiency and environmental impacts, although the adoption of CE is still limited. The study demonstrated that BIM integration with LCA can make great benefits in evaluating the whole-building life cycle, such as the quantification of materials with different alternatives, the selection of sustainable materials in the early design phase, and the faster and more accurate quantification and evaluation. CE adoption in BIM-based LCA of buildings is still limited, and lacks focus on whole building assessment in the existing studies. The circularity, especially the reusability and recyclability of the materials, and refurbishment and demolition process of a building are not comprehensively considered. In addition to identifying the existing challenges for CE adoption into BIM-based LCA of building, potential solutions along with a prospective framework are provided for LCA-based BIM design for sustainable buildings with evolving CE principles. This comprehensive framework is used to address the challenges of whole actors for achieving the decision-making process towards sustainability of future building.

Author Contributions: Conceptualization, M.U.H. and M.L.; methodology, M.U.H. and K.X.; investigation, M.M.; resources, M.H. and X.C.; writing-original draft preparation, K.X. and Y.Z.; writing-review and editing, M.U.H. and G.C.; funding acquisition, M.L. All authors have read and agreed to the published version of the manuscript.

Funding: This research was funded by the National Key R\&D Program of China of the 13th Five-Year (No. 2018YFD1100704). 
Institutional Review Board Statement: Not applicable.

Informed Consent Statement: Not applicable.

Data Availability Statement: Not applicable.

Acknowledgments: The authors acknowledge the support by National Centre for International Research of Low-carbon and Green Buildings and thank the China Scholarship Council (CSC) for sponsoring an academic visit at the Norwegian University of Science and Technology (NTNU) between 2019 and 2020.

Conflicts of Interest: The authors declare no conflict of interest.

\section{References}

1. Johnny, W.; Kuan, K.-L. Implementing 'BEAM Plus' for BIM-based sustainability analysis. Autom. Constr. 2014, $44,163-175$.

2. Anderson, J.E.; Wulfhorst, G.; Lang, W. Energy analysis of the built environment-A review and outlook. Renew. Sustain. Energy Rev. 2015, 44, 149-158. [CrossRef]

3. Antón, L.Á.; Díaz, J. Integration of Life Cycle Assessment in a BIM Environment. Procedia Eng. 2014, 85, 26-32. [CrossRef]

4. Jrade, A.; Jalaei, F. Integrating building information modelling with sustainability to design building projects at the conceptual stage. Build. Simul. 2013, 6, 429-444. [CrossRef]

5. Chong, W.K.; Kumar, S.; Haas, C.T.; Beheiry, S.; Coplen, L.; Oey, M. Understanding and Interpreting Baseline Perceptions of Sustainability in Construction among Civil Engineers in the United States. J. Manag. Eng. 2009, 25, 143-154. [CrossRef]

6. Wu, P.; Xia, B.; Wang, X. The contribution of ISO 14067 to the evolution of global greenhouse gas standards-A review. Renew. Sustain. Energy Rev. 2015, 47, 142-150. [CrossRef]

7. Soust-Verdaguer, B.; Llatas, C.; García-Martínez, A. Critical review of bim-based LCA method to buildings. Energy Build. 2017, 136, 110-120. [CrossRef]

8. Häkkinen, T.; Kiviniemi, A. Sustainable building and BIM. In Proceedings of the SB08 Conference Melbourne, Melbourne, Australia, 21-25 September 2008; pp. 21-25.

9. Mostavi, E.; Asadi, S.; Boussaa, D. Development of a new methodology to optimize building life cycle cost, environmental impacts, and occupant satisfaction. Energy 2017, 121, 606-615. [CrossRef]

10. Cavalliere, C.; Dell'Osso, G.R.; Pierucci, A.; Iannone, F. Life cycle assessment data structure for building information modelling. J. Clean. Prod. 2018, 199, 193-204. [CrossRef]

11. Peng, C. Calculation of a building's life cycle carbon emissions based on Ecotect and building information modeling. J. Clean. Prod. 2016, 112, 453-465. [CrossRef]

12. Schultz, J.; Ku, K.; Gindlesparger, M.; Doerfler, J. A benchmark study of BIM-based whole-building life-cycle assessment tools and processes. Int. J. Sustain. Build. Technol. Urban Dev. 2016, 7, 219-229. [CrossRef]

13. Russell-Smith, S.; Lepech, M. Activity-Based Methodology for Life Cycle Assessment of Building Construction. In Proceedings of the CIBSE ASHRAE Technical Symposium, London, UK, 18-19 April 2012.

14. Bribián, I.Z.; Aranda-Usón, A.; Scarpellini, S. Life cycle assessment in buildings: State-of-the-art and simplified LCA methodology as a complement for building certification. Build. Environ. 2009, 44, 2510-2520. [CrossRef]

15. Cavalliere, C.; Habert, G.; Dell'Osso, G.R.; Hollberg, A. Continuous BIM-based assessment of embodied environmental impacts throughout the design process. J. Clean. Prod. 2019, 211, 941-952. [CrossRef]

16. Hollberg, A.; Ruth, J. LCA in architectural design—A parametric approach. Int. J. Life Cycle Assess. 2016, 21, 943-960. [CrossRef]

17. Attia, S.; Gratia, E.; De Herde, A.; Hensen, J.L. Simulation-based decision support tool for early stages of zero-energy building design. Energy Build. 2012, 49, 2-15. [CrossRef]

18. Shi, X.; Yang, W. Performance-driven architectural design and optimization technique from a perspective of architects. Autom. Constr. 2013, 32, 125-135. [CrossRef]

19. Häkkinen, T.; Kuittinen, M.; Ruuska, A.; Jung, N. Reducing embodied carbon during the design process of buildings. J. Build. Eng. 2015, 4, 1-13. [CrossRef]

20. Azhar, S.; Carlton, W.A.; Olsen, D.; Ahmad, I. Building information modeling for sustainable design and LEED ${ }^{\circledR}$ rating analysis. Autom. Constr. 2011, 20, 217-224. [CrossRef]

21. Farzad Jalaei, A.J. An Automated BIM Model to Conceptually Design, Analyze, Simulate, and Assess Sustainable Building Projects. J. Constr. Eng. 2014, 2014, 672896. [CrossRef]

22. Röck, M.; Hollberg, A.; Habert, G.; Passer, A. LCA and BIM: Integrated Assessment and Visualization of Building Elements' Embodied Impacts for Design Guidance in Early Stages. Procedia CIRP 2018, 69, 218-223. [CrossRef]

23. Lu, Y.; Wu, Z.; Chang, R.; Li, Y. Building Information Modeling (BIM) for green buildings: A critical review and future directions. Autom. Constr. 2017, 83, 134-148. [CrossRef]

24. Ajayi, S.O.; Oyedele, L.; Ceranic, D.B.; Gallanagh, M.; Kadiri, K. Life cycle environmental performance of material specification: A BIM-enhanced comparative assessment. Int. J. Sustain. Build. Technol. Urban Dev. 2015, 6, 14-24. [CrossRef]

25. Wiberg, A.A.M.H.; Georges, L.; Dokka, T.H.; Haase, M.; Time, B.; Lien, A.G.; Mellegård, S.E.; Maltha, M.M. A net zero emission concept analysis of a single-family house. Energy Build. 2014, 74, 101-110. [CrossRef] 
26. Schwartz, Y.; Eleftheriadis, S.; Raslan, R.; Mumovic, D. Semantically Enriched BIM Life Cycle Assessment to Enhance Buildings' Environmental Performance. In Proceedings of the CIBSE Technical Symposium, Edinburgh, UK, 14-15 April 2016.

27. Dieterle, M.; Schäfer, P.; Viere, T. Life Cycle Gaps: Interpreting LCA Results with a Circular Economy Mindset. Procedia CIRP 2018, 69, 764-768. [CrossRef]

28. Geissdoerfer, M.; Savaget, P.; Bocken, N.M.P.; Hultink, E.J. The Circular Economy-A new sustainability paradigm? J. Clean. Prod. 2017, 143, 757-768. [CrossRef]

29. Akanbi, L.A.; Oyedele, L.O.; Akinade, O.O.; Ajayi, A.O.; Delgado, M.D.; Bilal, M.; Bello, S.A. Salvaging building materials in a circular economy: A BIM-based whole-life performance estimator. Resour. Conserv. Recycl. 2018, 129, 175-186. [CrossRef]

30. Herczeg, G.; Akkerman, R.; Hauschild, M.Z. Supply chain collaboration in industrial symbiosis networks. J. Clean. Prod. 2018, 171, 1058-1067. [CrossRef]

31. Ghisellini, P.; Ripa, M.; Ulgiati, S. Exploring environmental and economic costs and benefits of a circular economy approach to the construction and demolition sector. A literature review. J. Clean. Prod. 2018, 178, 618-643. [CrossRef]

32. Hossain, M.U.; Ng, S.T. Critical consideration of buildings' environmental impact assessment towards adoption of circular economy: An analytical review. J. Clean. Prod. 2018, 205, 763-780. [CrossRef]

33. Winans, K.; Kendall, A.; Deng, H. The history and current applications of the circular economy concept. Renew. Sustain. Energy Rev. 2017, 68, 825-833. [CrossRef]

34. Mckinsey \& Company. Towards the Circular Economy: Accelerating the Scale-Up across Global Supply Chains. 2014. Available online: http:/ / www3.weforum.org/docs/WEF_ENV_CircularEconomy_MainStream_ProjectOutline_2014.pdf (accessed on 23 May 2019).

35. Adams, K.T.; Osmani, M.; Thorpe, T.; Thornback, J. Circular economy in construction: Current awareness, challenges and enablers. Proc. Inst. Civ. Eng. Waste Resour. Manag. 2017, 170, 15-24. [CrossRef]

36. Russell-Smith, S.V.; Lepech, M.D. Cradle-to-gate sustainable target value design: Integrating life cycle assessment and construction management for buildings. J. Clean. Prod. 2015, 100, 107-115. [CrossRef]

37. Russell-Smith, S.V.; Lepech, M.D.; Fruchter, R.; Meyer, Y.B. Sustainable target value design: Integrating life cycle assessment and target value design to improve building energy and environmental performance. J. Clean. Prod. 2015, 88, 43-51. [CrossRef]

38. Liu, M.; Li, B.; Yao, R.-M. Embodied coefficient of energy carriers and its calculation method. J. Cent. South Univ. Technol. 2011, 18, 1293-1298. [CrossRef]

39. Azhar, S.; Brown, J. BIM for Sustainability Analyses. Int. J. Constr. Educ. Res. 2009, 5, 276-292. [CrossRef]

40. Soust-Verdaguer, B.; Llatas, C.; García-Martínez, A. Simplification in life cycle assessment of single-family houses: A review of recent developments. Build. Environ. 2016, 103, 215-227. [CrossRef]

41. Basbagill, J.P.; Flager, F.L.; Lepech, M.D.; Fischer, M. Application of life-cycle assessment to early stage building design for reduced embodied environmental impacts. Build. Environ. 2013, 60, 81-92. [CrossRef]

42. Santos, R.; Costa, A.A. BIM in LCA/LCEA Analysis: Comparative analysis of Multi-family House and Single-family. In Proceedings of the CIB World Building Congress, Tampere, Finland, 30 May-3 June 2016.

43. Lee, S.; Tae, S.; Roh, S.; Kim, T. Green Template for Life Cycle Assessment of Buildings Based on Building Information Modeling: Focus on Embodied Environmental Impact. Sustainability 2015, 7, 16498-16512. [CrossRef]

44. Ramaji, I.J.; Gultekin-Bicer, P.; Crowley, R.W.; Lambert, J.D. Investigation of Leveraging BIM Standards to Facilitate Sustainability Evaluations from Early Stages of Design. In Computing in Civil Engineering 2017; American Society of Civil Engineers (ASCE): Reston, VA, USA, 2017; Volume 2017, pp. 175-183.

45. Wang, W.; Zmeureanu, R.; Rivard, H. Applying multi-objective genetic algorithms in green building design optimization. Build. Environ. 2005, 40, 1512-1525. [CrossRef]

46. Kulahcioglu, T.; Dang, J.B.; Toklu, C. A 3D analyzer for BIM-enabled Life Cycle Assessment of the whole process of construction. HvacER Res. 2012, 18, 283-293.

47. Mao, C.; Shen, Q.; Wang, J.; Lu, C. A BIM-enabled Estimating Framework of Embodied Emissions of Building Materials for Optimization in Design Stage: A Case Study. Int. J. Digit. Content Technol. Appl. 2013, 7, 645-653. [CrossRef]

48. Wang, E.; Shen, Z.; Berryman, C. A Building LCA Case Study Using Autodesk Ecotect and BIM Model. In Proceedings of the 47th ASC Annual International Conference, Omaha, NE, USA, 6-9 April 2011.

49. Crippa, J.; Boeing, L.C.; Caparelli, A.P.A.; Costa, M.D.R.D.M.M.D.; Scheer, S.; Araujo, A.M.F.; Bem, D. A BIM-LCA integration technique to embodied carbon estimation applied on wall systems in Brazil. Built Environ. Proj. Asset Manag. 2018, 8, 491-503. [CrossRef]

50. Soust-Verdaguer, B.; Llatas, C.; García-Martínez, A.; De Cózar, J.C.G. BIM-Based LCA Method to Analyze Envelope Alternatives of Single-Family Houses: Case Study in Uruguay. J. Arch. Eng. 2018, 24, 05018002. [CrossRef]

51. Bueno, C.; Fabricio, M.M. Comparative analysis between a complete LCA study and results from a BIM-LCA plug-in. Autom. Constr. 2018, 90, 188-200. [CrossRef]

52. Yang, X.; Hu, M.; Wu, J.; Zhao, B. Building-information-modeling enabled life cycle assessment, a case study on carbon footprint accounting for a residential building in China. J. Clean. Prod. 2018, 183, 729-743. [CrossRef]

53. Chong, H.-Y.; Lee, C.-Y.; Wang, X. A mixed review of the adoption of Building Information Modelling (BIM) for sustainability. J. Clean. Prod. 2017, 142, 4114-4126. [CrossRef] 
54. Eleftheriadis, S.; Mumovic, D.; Greening, P. Life cycle energy efficiency in building structures: A review of current developments and future outlooks based on BIM capabilities. Renew. Sustain. Energy Rev. 2017, 67, 811-825. [CrossRef]

55. Kylili, A.; Fokaides, P.A.; Vaiciunas, J.; Seduikyte, L. Integration of Building Information Modelling (BIM) and Life Cycle Assessment (LCA) for sustainable constructions. J. Sustain. Arch. Civ. Eng. 2016, 13, 28-38. [CrossRef]

56. Wong, J.K.W.; Zhou, J. Enhancing environmental sustainability over building life cycles through green BIM: A review. Autom. Constr. 2015, 57, 156-165. [CrossRef]

57. Kreiner, H.; Passer, A.; Wallbaum, H. A new systemic approach to improve the sustainability performance of office buildings in the early design stage. Energy Build. 2015, 109, 385-396. [CrossRef]

58. Huali, W. Application Research of BIM in Environment Assessment of Full Life Cycle for Construction Projects. In Proceedings of the 2017 International Conference on Smart Grid and Electrical Automation (ICSGEA), Changsha, China, 27-28 May 2017; pp. 652-655.

59. Liu, M.; Li, B.; Yao, R. A generic model of Exergy Assessment for the Environmental Impact of Building Lifecycle. Energy Build. 2010, 42, 1482-1490. [CrossRef]

60. Najjar, M.; Figueiredo, K.; Palumbo, M.; Haddad, A. Integration of BIM and LCA: Evaluating the environmental impacts of building materials at an early stage of designing a typical office building. J. Build. Eng. 2017, 14, 115-126. [CrossRef]

61. Institute, S.M. Athena EcoCalcultor for Commercial Assemblies. Version 3.6. 2011. Available online: https://calculatelca.com/ software/ecocalculator/ (accessed on 23 May 2019).

62. Dallas: Beck Technology, L. DProfiler. Version 2.0. 2012. Available online: https://beck-technology.com/category/destiniprofiler/ (accessed on 23 May 2019).

63. Phoenix Integration, ModelCenter. Version 10.0. 2008. Available online: https://www.phoenix-int.com/product/modelcenterintegrate/ (accessed on 23 May 2019).

64. Garcia, D. Cost Performance Based Design-Using Digital Technology for Cost Performance Simulation in the Conceptual Phase of Design. 2015. Available online: http://papers.cumincad.org/data/works/att/ecaade2015_306.content.pdf (accessed on 23 May 2019).

65. James, J. Hirsch \& Associates. eQUEST. Version 3.64. 2010. Available online: http://www.doe2.com/eQUEST (accessed on 23 May 2019).

66. Werner, F.; Richter, K. Wooden building products in comparative LCA. Int. J. Life Cycle Assess. 2007, 12, 470-479.

67. Zhu, D. Circular economy: New economy for 21 century. Empir. Ref. 2005, 8, 28-30.

68. Mitchell, P.; Morgan, J. Employment and the Circular Economy Job Creation in a More Resource Efficient Britain; Green Alliance: London, $\mathrm{UK}, 2015$.

69. Su, B.; Heshmati, A.; Geng, Y.; Yu, X. A review of the circular economy in China: Moving from rhetoric to implementation. J. Clean. Prod. 2013, 42, 215-227. [CrossRef]

70. Nasir, M.H.A.; Genovese, A.; Acquaye, A.A.; Koh, S.; Yamoah, F. Comparing linear and circular supply chains: A case study from the construction industry. Int. J. Prod. Econ. 2017, 183, 443-457. [CrossRef]

71. Pomponi, F.; Moncaster, A. Circular economy for the built environment: A research framework. J. Clean. Prod. 2017, 143, 710-718. [CrossRef]

72. Dadhich, P.; Genovese, A.; Kumar, N.; Acquaye, A. Developing sustainable supply chains in the UK construction industry: A case study. Int. J. Prod. Econ. 2015, 164, 271-284. [CrossRef]

73. Leising, E.; Quist, J.; Bocken, N. Circular Economy in the building sector: Three cases and a collaboration tool. J. Clean. Prod. 2018, 176, 976-989. [CrossRef]

74. De Jesus, A.; Antunes, P.; Santos, R.; Mendonça, S. Eco-innovation in the transition to a circular economy: An analytical literature review. J. Clean. Prod. 2018, 172, 2999-3018. [CrossRef]

75. Korhonen, J.; Honkasalo, A.; Seppälä, J. Circular Economy: The Concept and its Limitations. Ecol. Econ. 2018, 143, 37-46. [CrossRef]

76. Gervásio, H.; Santos, P.; Martins, R.; Da Silva, L.S. A macro-component approach for the assessment of building sustainability in early stages of design. Build. Environ. 2014, 73, 256-270. [CrossRef]

77. Singh, J.; Ordoñez, I. Resource recovery from post-consumer waste: Important lessons for the upcoming circular economy. J. Clean. Prod. 2016, 134, 342-353. [CrossRef]

78. Pauliuk, S. Critical appraisal of the circular economy standard BS 8001:2017 and a dashboard of quantitative system indicators for its implementation in organizations. Resour. Conserv. Recycl. 2018, 129, 81-92. [CrossRef]

79. Haupt, M.; Vadenbo, C.; Hellweg, S. Do We Have the Right Performance Indicators for the Circular Economy?: Insight into the Swiss Waste Management System. J. Ind. Ecol. 2016, 21, 615-627. [CrossRef]

80. Sassanelli, C.; Rosa, P.; Rocca, R.; Terzi, S. Circular economy performance assessment methods: A systematic literature review. J. Clean. Prod. 2019, 229, 440-453. [CrossRef]

81. Braungart, M.; McDonough, W.; Bollinger, A. Cradle-to-cradle design: Creating healthy emissions-A strategy for eco-effective product and system design. J. Clean. Prod. 2007, 15, 1337-1348. [CrossRef]

82. Ploeger, H.; Prins, M.; Straub, A.; Brink, R.V.D. Circular economy and real estate: The legal (im)possibilities of operational lease. Facilities 2019, 37, 653-668. [CrossRef] 
83. Eberhardt, L.; Birgisdottir, H.; Birkved, M. Comparing life cycle assessment modelling of linear vs. circular building components. In Proceedings of the IOP Conference Series: Earth and Environmental Science; IOP Publishing: Bristol, UK, 2019; Volume 225, p. 012039.

84. Eadie, R.; Browne, M.; Odeyinka, H.; McKeown, C.; McNiff, S. BIM implementation throughout the UK construction project lifecycle: An analysis. Autom. Constr. 2013, 36, 145-151. [CrossRef]

85. Hossain, M.U.; Ng, S.T. Influence of waste materials on buildings' life cycle environmental impacts: Adopting resource recovery principle. Resour. Conserv. Recycl. 2019, 142, 10-23.

86. Hossain, U.; Wu, Z.; Poon, C.S. Comparative environmental evaluation of construction waste management through different waste sorting systems in Hong Kong. Waste Manag. 2017, 69, 325-335. [CrossRef] [PubMed]

87. Sanchez, B.; Rausch, C.; Haas, C. Deconstruction programming for adaptive reuse of buildings. Automat. Constr. 2019, $107,102921$. [CrossRef]

88. Potrč Obrecht, T.; Röck, M.; Hoxha, E.; Passer, A. BIM and LCA Integration: A Systematic Literature Review. Sustainability 2020, 12, 5534. [CrossRef]

89. Marrero, M.; Wojtasiewicz, M.; Martínez-Rocamora, A.; Solís-Guzmán, J.; Alba-Rodríguez, M.D. BIM-LCA Integration for the Environmental Impact Assessment of the Urbanization Process. Sustainability 2020, 12, 4196. [CrossRef]

90. Naneva, A.; Bonanomi, M.; Hollberg, A.; Habert, G.; Hall, D.M. Integrated BIM-Based LCA for the Entire Building Process Using an Existing Structure for Cost Estimation in the Swiss Context. Sustainability 2020, 12, 3748. [CrossRef]

91. Santos, R.; Costa, A.A.; Silvestre, J.D.; Pyl, L. Development of a BIM-based Environmental and Economic Life Cycle Assessment tool. J. Clean. Prod. 2020, 265, 121705. [CrossRef]

92. Mora, T.D.; Bolzonello, E.; Cavalliere, C.; Peron, F. Key Parameters Featuring BIM-LCA Integration in Buildings: A Practical Review of the Current Trends. Sustainability 2020, 12, 7182. [CrossRef]

93. Kalmykova, Y.; Sadagopan, M.; Rosado, L. Circular economy-From review of theories and practices to development of implementation tools. Resour. Conserv. Recycl. 2018, 135, 190-201. [CrossRef]

94. Santos, R.; Costa, A.A.; Silvestre, J.D.; Pyl, L. Informetric analysis and review of literature on the role of BIM in sustainable construction. Autom. Constr. 2019, 103, 221-234. [CrossRef]

95. Manninen, K.; Koskela, S.; Antikainen, R.; Bocken, N.; Bontempi, E.; Aminoff, A. Do circular economy business models capture intended environmental value propositions? J. Clean. Prod. 2018, 171, 413-422. [CrossRef] 